 \\ Hif \\ HSOA Journal of HЕRАD Pulmonary Medicine \& Respiratory Research
}

\section{Organising Pneumonia Sec- ondary to 6-Mercaptopurine in a Patient with Inflammatory Bowel Disease}

\author{
Ahmed Fahim*and Laura Jones \\ Department of Respiratory Medicine, McHale Centre, New Cross Hospital, \\ Wolverhampton, UK
}

\begin{abstract}
A 45 year old woman with a background of ulcerative colitis presented with a history of non-productive cough and progressive shortness of breath that failed to respond to antibiotics. She had been commenced on 6-Mercaptopurine (6-MP) for Inflammatory Bowel Disease (IBD) six weeks prior to developing respiratory symptoms. Laboratory and radiological investigations suggested a pneumonic process that failed to resolve following broad-spectrum intravenous antibiotics. A thoracoscopic biopsy demonstrated features consistent with organising pneumonia, which was felt to be secondary to 6-MP as her symptoms coincided following the introduction of the drug. There was complete resolution of pneumonic changes following cessation of 6-MP. This case highlights the importance of considering 6-MP as a potential cause of organising pneumonia in appropriate clinical context.

Keywords: 6-mercaptopurine; Organising pneumonia; Ulcerative colitis
\end{abstract}

\section{Introduction}

Organising pneumonia is characterised by histopathological evidence of intra-alveolar fibroblastic plugs and uni-or multi-focal consolidation in a bronchovascular distribution on thoracic imaging. There is no gender predilection and it usually presents in the fifth decade. The most common clinical presentation is of a non-resolving lower respiratory tract infection with symptoms of shortness of breath, non-productive cough and malaise. A careful history is invaluable to determine if the Organising Pneumonia is Cryptogenic (COP) or if it is secondary to infection, medication or associated with another underlying condition such as a connective tissue disease. The aetiology of organising pneumonia is diverse (Table 1) and the key to diagnosis is a thorough clinical history that includes exposure to any potential drugs implicated in the causation of this interstitial lung disease.

*Corresponding author: Ahmed Fahim, Department of Respiratory Medicine, McHale Centre, New Cross Hospital, West Midlands WV10 OQP, UK, Tel: +44 1902694106; E-mail: ahmedfahim@doctors.org.uk

Citation: Fahim A, Jones $L$ (2016) Organising Pneumonia Secondary to 6-Mercaptopurine in a Patient with Inflammatory Bowel Disease. J Pulm Med Respir Res 2: 005

Received: April 13, 2016; Accepted: May 10, 2016; Published: May 24, 2016

\begin{tabular}{|l|l|}
\hline \multicolumn{2}{|c|}{ Causes of organising pneumonia } \\
\hline & Infections (Bacterial, Viral, Fungal or Parasitic) \\
\cline { 1 - 2 } Causes & Connective tissue diseases \\
\cline { 2 - 3 } & Inflammatory bowel disease \\
\cline { 2 - 3 } & Aspiration \\
\cline { 2 - 3 } & Radiation \\
\cline { 2 - 3 } & Haematological malignancies \\
\cline { 2 - 3 } & (NSIP) Non Specific Interstitial Pneumonia \\
\cline { 2 - 3 } & Immunodeficiency \\
\cline { 2 - 2 } & Drugs \\
\hline
\end{tabular}

\section{Case Report}

We report a case of a 45-year old woman who developed an organising pneumonia secondary to 6-Mercaptopurine (6-MP). She had a background history of pan-ulcerative colitis diagnosed 7 years ago. It was treated with oral corticosteroids and mesalazine followed by the addition of azathioprine as a steroid sparing agent. However, she failed to tolerate azathioprine due to severe lethargy.She was a lifelong non-smoker and had no other co-morbidities.

She was commenced on 6-MP 50mg once daily as an alternative immunosuppressant to azathioprine. However, she developed a non-productive cough with shortness of breath after 6 weeks of commencing 6-MP. She was treated for a lower respiratory tract infection with a couple of antibiotic courses (amoxicillin 500mg TDS followed by clarithromycin 500mg BD for one week each) by general practitioner. Unfortunately, she failed to respond to antibiotics and was referred to acute medical unit.

On examinationshe was hypoxic with oxygen saturation of $93 \%$ on breathing room air. A chest radiograph showed predominantly left sided consolidation (Figure 1) with volume loss. Blood work up revealed raised inflammatory markers (C-reactive protein 167) so she was treated for a community acquired pneumonia with intravenous Tazocin (Tazobactam and Piperacillin) 4.5g TDS. Subsequent blood cultures, atypical pneumonia screen and HIV antibody was negative. A flexible fiberoptic bronchoscopy (Olympus BF-160) was carried out to obtain samples for detailed microbiological testing and atypical organisms including mycobacterial infection. Bronchial washings did not reveal any positive evidence of infective process. A repeat chest radiograph 6 weeks after the initial presentation showed worsening pulmonary infiltrates (Figure 2). Hence, a High Resolution Thoracic CT Scan (HRCT) was arranged which revealed bilateral consolidation with volume loss and associated traction bronchiectasis suggesting a fibrotic process (Figure 3). Pulmonary function tests demonstrated mild restriction.

As there was a poor response to antibiotics with persistent parenchymal infiltrates, a Video Assisted Thoracoscopic Biopsy (VATs) was obtained which showed patchy interstitial pneumonitis with resolving organising pneumonia (Figure 4). This was suggestive of drug induced pneumonitis/organising pneumonia. Following discussion at interstitial lung disease meeting it was felt that 
Citation: Fahim A, Jones L (2016) Organising Pneumonia Secondary to 6-Mercaptopurine in a Patient with Inflammatory Bowel Disease. J Pulm Med Respir Res 2: 005.

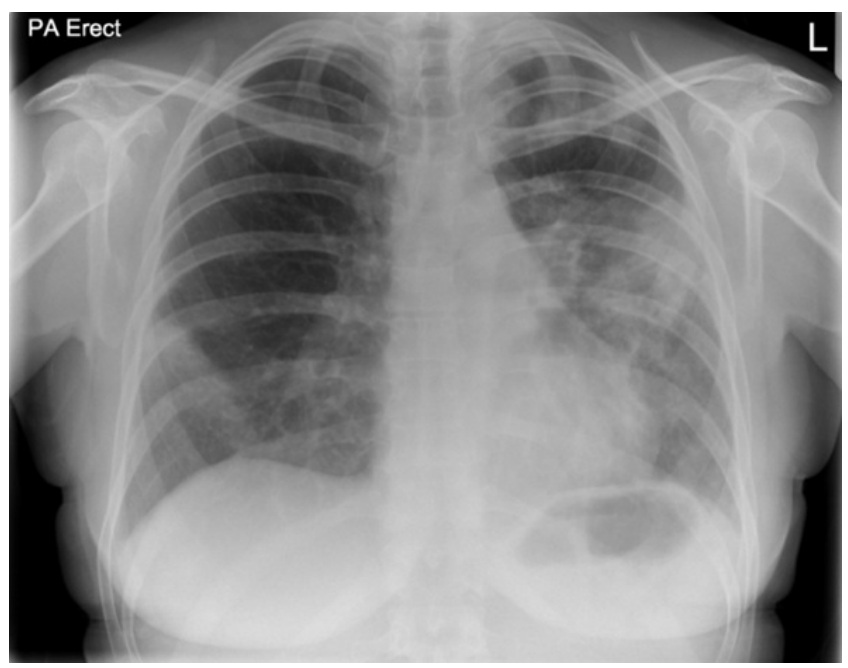

Figure 1: Chest radiograph showing predominantly left sided peripheral consolidation.

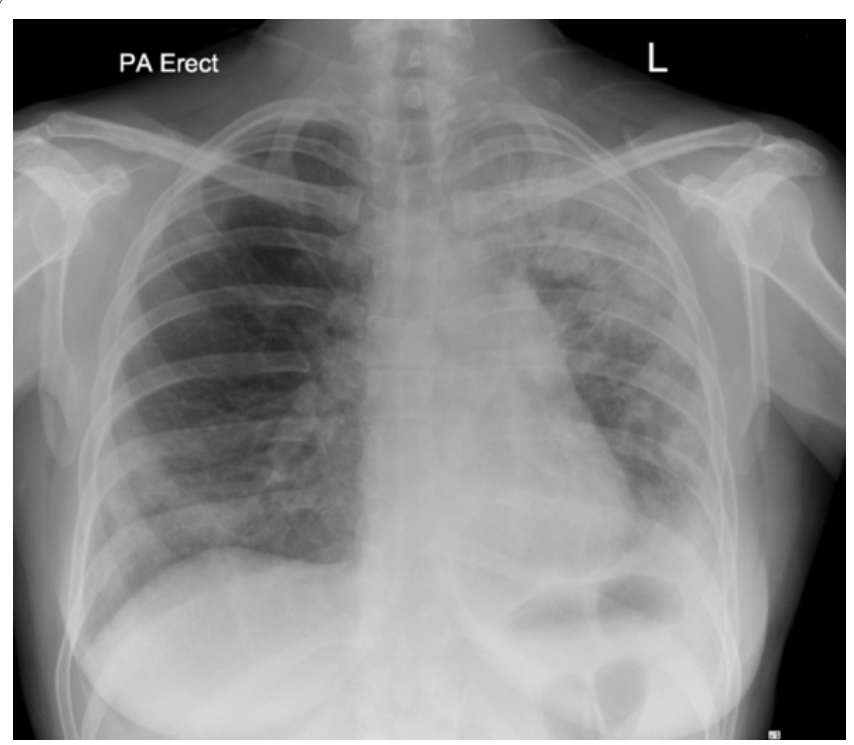

Figure 2: A repeat chest radiograph 6 weeks after the initial presentation shows worsening consolidation on left side.

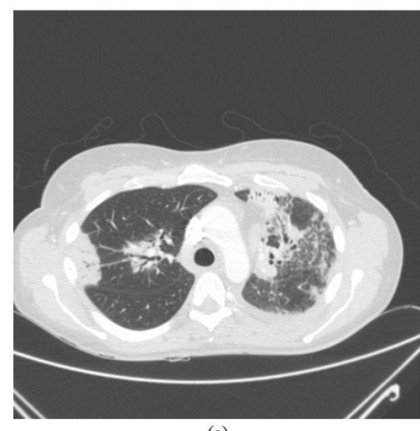

(a)

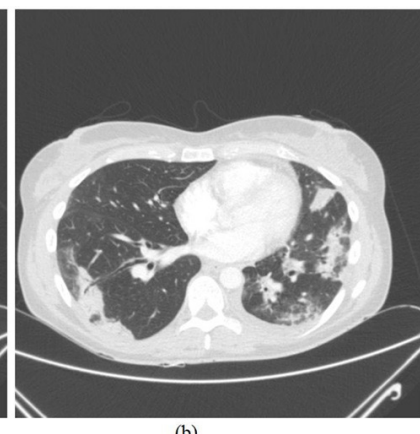

(b)
Figure 3: (a) CT thorax at the level of upper lobes demonstrating bilateral consolidation in a peribronchovascular distribution with ground glass change associated with it. This radiological picture is highly suggestive of organising pneumonia. (b) Similar findings to figure 3 (a) but at a lower cross sectional level of thorax. the clinico-radiological picture, coupled with lung biopsy, was consistent with organising pneumonia secondary to 6-MP. With the cessation of 6-MP, the patient made an excellent recovery and radiological abnormality resolved completely 4 months after the initial presentation (Figure 5) and patient is well at 18 months of follow up.

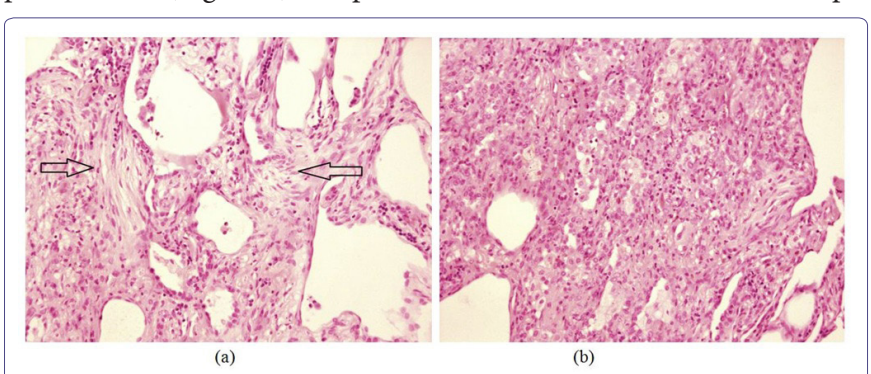

Figure 4: (a) Video Assisted Thoracoscopic (VATS) lung biopsy showing resolving organising pneumonia. There is evidence of granulation tissue with fibroblastic plugs in the biopsy specimen (arrows). (b) Lung biopsy demonstrating inflammatory cell infiltrate including eosinophils and lymphocytes

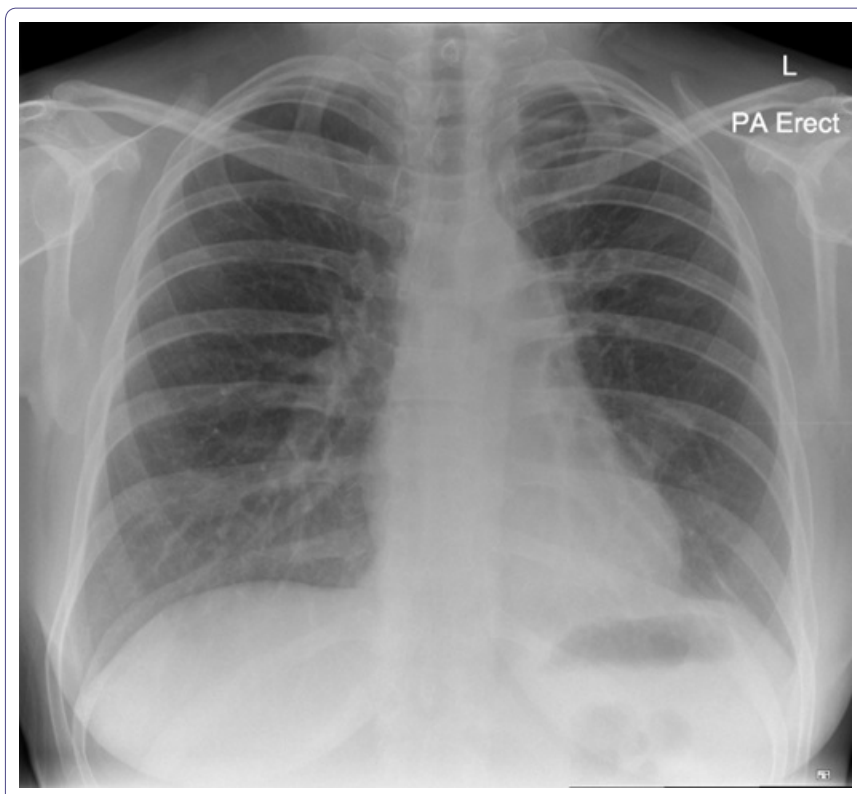

Figure 5: Follow up chest radiograph showing complete resolution of parenchymal infiltrates.

\section{Discussion}

Organising pneumonia is a distinct disease entity that usually presents with progressive shortness of breath and should be considered in a patient who fails to respond to antibiotic treatment. It can be divided into three categories dependent on the aetiology $[1,2]$; organising pneumonia of determined cause, Organising Pneumonia of unknown cause or Cryptogenic (COP) and organising pneumonia of undetermined cause but occurring in a specific clinical context such as granulomatous disease or vasculitis. Our case had a background of inflammatory bowel disease which is well recognised to have an association with a variety of lung pathologies including organising pneumonia. It was felt, however that given the timing of onset of symptoms and the improvement on cessation of the 6-MP that this was the cause of the organising pneumonia.

Organising pneumonia or Bronchiolitis Obliterans Organising Pneumonia (BOOP) was originally described as a failure of 
resolution of typical acute lobar pneumonia and the pathological hallmark of organising pneumonia i.e., Masson bodies were described by Masson and colleagues in association with rheumatoid arthritis. It is an interstitial lung disease with generally a favourable prognosis and may resolve spontaneously. It is generally recommended to commence corticosteroids in organising pneumonia; particularly when it is idiopathic (COP), however, this recommendation is not supported by high quality randomised clinical trials and is based on observational and retrospective studies [3-6]. The decision to commence corticosteroids in cases of organising pneumonia should be based on individual patient characteristics and a frank discussion with a well-informed patient taking into account the potential adverse effects of high dose corticosteroid use and the clinical benefit likely to be achievable.

Thiopurine analogues such as azathioprine and 6-MP are commonly prescribed in the treatment of Inflammatory Bowel Disease (IBD) as immunomodulating drugs. These drugs are used as maintenance therapy as well as steroid sparing agents in IBD. A variety of different toxic pulmonary patterns secondary to azathioprine have been reported in literature including interstitial pneumonitis [7], pulmonary fibrosis and pulmonary oedema [8]. However, there is paucity of data regarding 6-MP induced pulmonary toxicity. Ananthakrishnan et al., [9] reported 3 cases of severe non-infectious pulmonary toxicity within one month of commencing azathioprine/6-MP for IBD. Histopathological examination revealed BOOP and Usual Interstitial Pneumonia (UIP) and all 3 patients improved after the cessation of therapy. Our patient had slightly delayed symptoms as compared to this case series and one of the patients in this series had similar histological profile as described in our case (i.e., BOOP).

\section{Mechanism of pulmonary toxicity by thiopurines}

Although the exact mechanism of 6-MP related pulmonary parenchymal injury is unknown, we believe it is due to immunologically mediated mechanisms from the drug and its metabolites. A variety of mechanisms of pulmonary alveolar injury may be responsible as there are multiple patterns of toxicity seen due to these drugs. In our opinion, the most likely mechanism in 6-MP induced lung injury is combination of alveolitis and pulmonary oedema followed by impaired tissue repair that leads to the formation of granulation and fibrotic tissue. Furthermore, hypersensitivity reaction in the form of pneumonitis accompanies the subsequent changes seen in pulmonary interstitium. The initial inflammation is linked with direct effects of 6-MP to pulmonary tissue and subsequent aberrant repair led to organising pneumonia development that responded to cessation of therapy with thiopurine. It is important to acknowledge that actual frequency of pulmonary toxicity due to 6-MP is unknown and reporting similar cases to ours will highlight the scope of the problem and help elucidate underlying pathogenetic mechanisms involved in the development of pneumonitis and organising pneumonia.

\section{Key Points}

This is a rare association between 6-MP and the development of organising pneumonia. Although organising pneumonia is a relatively rare condition it will often present to the general and acute physicians as a non-resolving pneumonia or lower respiratory tract infection. It is important for general, respiratory and acute physicians and gastroenterologists to be aware of the association described. Moreover, this case highlights the importance of thorough clinical history and clinicians having a high index of suspicion to identify potential cases of 6-MP related organising pneumonia and pneumonitis that may have been under-recognised and under reported.

\section{Conflicts of Interests}

None to declare for any of the authors in relation to this manuscript

\section{Patient Consent}

Obtained

\section{References}

1. Cordier JF (2006) Cryptogenic organising pneumonia. Eur Respir J 28: 422446.

2. Cordier JF (2000) Organising pneumonia. Thorax 55: 318-328.

3. Davison AG, Heard BE, McAllister WA, Turner-Warwick ME (1983) Cryptogenic organizing pneumonitis. Q J Med 52: 382-394.

4. Epler GR (2001) Bronchiolitis obliterans organizing pneumonia. Arch Intern Med 161: 158-164.

5. Cazzato S, Zompatori M, Baruzzi G, Schiattone ML, Burzi M et al. (2000) Bronchiolitis obliterans-organizing pneumonia: an Italian experience. Respir Med 94: 702-708.

6. Alasaly K, Muller N, Ostrow DN, Champion P, FitzGerald JM (1995) Cryptogenic organizing pneumonia: A report of 25 cases and a review of the literature. Medicine (Baltimore) 74: 201-211.

7. Nagy F, Molnar T, Makula E, Kiss I, Milassin P, et al. (2007) A case of interstitial pneumonitis in a patient with ulcerative colitis treated with azathioprine. World J Gastroenterol 13: 316-319.

8. Fauroux B, Meyer-Milsztain A, Boccon-Gibod L, Leverger G, Clément A, et al. (1994) Cytotoxic drug-induced pulmonary disease in infants and children. Pediatr Pulmonol 18: 347-355.

9. Ananthakrishnan AN, Attila T, Otterson MF, Lipchik RJ, Massey BT, et al. (2007) Severe pulmonary toxicity after azathioprine/6-mercaptopurine initiation for the treatment of inflammatory bowel disease. J Clin Gastroenterol 41: 682-688. 